\title{
Differential Site-Based Expression of Pentose Phosphate Pathway-Related Proteins among Breast Cancer Metastases
}

\author{
Yoon Jin Cha, ${ }^{1}$ Woo Hee Jung, ${ }^{1}$ and Ja Seung Koo ${ }^{2}$ \\ ${ }^{1}$ Department of Pathology, Gangnam Severance Hospital, Yonsei University College of Medicine, 211 Eonju-ro, \\ Gangnam-gu, Seoul 06273, Republic of Korea \\ ${ }^{2}$ Department of Pathology, Severance Hospital, Yonsei University College of Medicine, 50-1 Yonsei-ro, Seodaemun-gu, \\ Seoul 03722, Republic of Korea \\ Correspondence should be addressed to Ja Seung Koo; kjs1976@yuhs.ac
}

Received 11 November 2016; Revised 22 December 2016; Accepted 15 January 2017; Published 2 February 2017

Academic Editor: Szilárd Nemes

Copyright (C) 2017 Yoon Jin Cha et al. This is an open access article distributed under the Creative Commons Attribution License, which permits unrestricted use, distribution, and reproduction in any medium, provided the original work is properly cited.

Purpose. We aimed to investigate the expression of pentose phosphate pathway- (PPP-) related proteins in metastatic breast cancer and its relationship with clinicopathologic factors. Methods. Tissue samples from 126 metastatic breast cancers were included in a tissue microarray. Expression of PPP-related proteins [glucose-6-phosphate dehydrogenase (G6PDH), 6-phosphogluconolactonase (6PGL), 6-phosphogluconate dehydrogenase (6PGDH), and nuclear factor erythroid 2-related factor (NRF2)] was determined by immunohistochemistry. Results. G6PDH $(p=0.011)$ and cytoplasmic NRF2 $(p=0.001)$ showed the highest expression in brain metastases. Human epidermal growth factor receptor (HER-2) positivity was associated with G6PDH $(p<0.001)$ and cytoplasmic NRF2 ( $p=0.015)$ positivity. A high Ki-67 labeling index (LI) was correlated with nuclear NRF2 positivity $(p=0.037)$, and HER-2positive luminal B type was associated with G6PDH positivity $(p=0.001)$. On multivariate Cox analysis, independent risk factors of short overall survival were 6PGL positivity in bone metastasis (HR 4.180, 95\% CI 1.160-15.06, $p=0.029$ ) and low Ki-67 LI in lung metastasis (HR 11.853, 95\% CI 1.841-76.30, $p=0.009$ ). Conclusion. Differential expression of PPP-related proteins correlated with different prognoses and metastatic sites, with the highest expression in brain metastases, and could be a potential therapeutic target.

\section{Introduction}

The pentose phosphate pathway (PPP) is a metabolic pathway parallel to glycolysis. The PPP links glucose metabolism with ribose production and NADPH generation. The PPP comprises oxidative and nonoxidative branches. The oxidative branch generates NADPH and ribonucleotides, with enzymatic regulation by glucose-6-phosphate dehydrogenase (G6PDH), 6-phosphogluconolactonase (6PGL), and 6phosphogluconate dehydrogenase (6PGDH). Most of the pentose phosphate in the body, which is required in rapidly proliferative cells, is derived from the PPP. In cancer cells, the PPP generates pentose phosphate as well as NADPH, which is important in lipid synthesis and cell survival under stressful conditions. Thus, the PPP plays a pivotal role in constantly proliferating cancer cells, and increased expression of PPPrelated enzymes in cancer tissue has been reported [1-3].
Breast cancer has high morbidity and mortality rates, caused by distant metastasis of primary tumors. Breast cancer commonly metastasizes to the lung, brain, liver, and bone $[4,5]$, and brain and bone metastases have been thoroughly investigated [6-11]. Tumor metastasis generally occurs by reciprocal interaction between tumor cells and host tissue via adhesion, proteolysis, invasion, and angiogenesis [4, 12]. Because not all tumors have similar metastatic patterns, the seed and soil hypothesis was proposed to explain tumor metastasis as the survival of a specific tumor (seed) in a specific visceral organ (soil) [13]. Breast cancer metastases have different signatures according to the metastatic sites. Brain metastases have specific clinical characteristics such as young patient age, estrogen receptor (ER) negativity, prior lung metastasis, human epidermal growth factor receptor(HER-) 2 amplification, epidermal growth factor receptor (EGFR) overexpression, and basal subtype $[8,9,11]$. In 
TABLE 1: Source, clone, and dilution of antibodies.

\begin{tabular}{|c|c|c|c|}
\hline Antibody & Company & Clone & Dilution \\
\hline \multicolumn{4}{|c|}{$\begin{array}{l}\text { Pentose phosphate pathway-related } \\
\text { proteins }\end{array}$} \\
\hline G6PDH & Abcam, Cambridge, UK & Polyclonal & $1: 100$ \\
\hline $6 \mathrm{PGL}$ & Abcam, Cambridge, UK & ERP1238(B) & $1: 200$ \\
\hline $6 \mathrm{PGDH}$ & Abcam, Cambridge, UK & Polyclonal & $1: 100$ \\
\hline NRF2 & Abcam, Cambridge, UK & Polyclonal & $1: 50$ \\
\hline \multicolumn{4}{|c|}{ Molecular subtype related proteins } \\
\hline ER & Thermo Scientific, San Diego, CA, USA & SP1 & $1: 100$ \\
\hline PR & DAKO, Glostrup, Denmark & PgR & $1: 50$ \\
\hline HER-2 & DAKO, Glostrup, Denmark & Polyclonal & $1: 1500$ \\
\hline $\mathrm{Ki}-67$ & Abcam, Cambridge, UK & MIB & $1: 1000$ \\
\hline
\end{tabular}

G6PDH, glucose-6-phosphate dehydrogenase; 6PGL, 6-phosphogluconolactonase; 6PGDH, 6-phosphogluconate dehydrogenase; NRF2, nuclear factor erythroid 2- (NF-E2-) related factor 2; ER, estrogen receptor; PR, progesterone receptor; HER-2, human epidermal growth factor-2.

TABLE 2: Basal characteristics of patients with metastatic breast cancer.

\begin{tabular}{|c|c|c|c|c|c|c|}
\hline \multirow{2}{*}{ Parameter } & \multirow{2}{*}{$\begin{array}{c}\text { Total } \\
N=126(\%)\end{array}$} & \multicolumn{4}{|c|}{ Metastatic site } & \multirow{2}{*}{$p$ value } \\
\hline & & $\begin{array}{c}\text { Bone } \\
N=31(\%)\end{array}$ & $\begin{array}{c}\text { Brain } \\
N=36(\%)\end{array}$ & $\begin{array}{c}\text { Liver } \\
N=11(\%)\end{array}$ & $\begin{array}{c}\text { Lung } \\
N=48(\%)\end{array}$ & \\
\hline \multicolumn{7}{|l|}{ Age (years) } \\
\hline$\leq 50$ & $65(51.6)$ & $17(54.8)$ & $17(47.2)$ & $4(36.4)$ & $27(56.3)$ & \multirow{2}{*}{0.605} \\
\hline$>50$ & $61(48.4)$ & $14(45.2)$ & $19(52.8)$ & 7 (63.6) & $21(43.8)$ & \\
\hline \multicolumn{7}{|l|}{ ER } \\
\hline Negative & $59(46.8)$ & $6(19.4)$ & $25(69.4)$ & $2(18.2)$ & $26(54.2)$ & \multirow{2}{*}{$<0.001$} \\
\hline Positive & $67(53.2)$ & $25(80.6)$ & $11(30.6)$ & $9(81.8)$ & $22(45.8)$ & \\
\hline \multicolumn{7}{|l|}{ PR } \\
\hline Negative & $86(68.3)$ & $16(51.6)$ & $35(97.2)$ & $3(27.3)$ & $32(66.7)$ & \multirow{2}{*}{$<0.001$} \\
\hline Positive & $40(31.7)$ & $15(48.4)$ & $1(2.8)$ & $8(72.7)$ & $16(33.3)$ & \\
\hline \multicolumn{7}{|l|}{ HER-2 } \\
\hline Negative & $86(68.3)$ & $25(80.6)$ & $18(50.0)$ & $9(81.8)$ & $34(70.8)$ & \multirow{2}{*}{0.032} \\
\hline Positive & $40(31.7)$ & $6(19.4)$ & $18(50.0)$ & $2(18.2)$ & $14(29.2)$ & \\
\hline \multicolumn{7}{|l|}{ Ki-67 LI } \\
\hline$<14$ & $84(66.7)$ & $27(87.1)$ & $18(50.0)$ & $9(81.8)$ & $30(62.5)$ & \multirow{2}{*}{0.008} \\
\hline$\geq 14$ & $42(33.3)$ & $4(12.9)$ & $18(50.0)$ & $2(18.2)$ & $18(37.5)$ & \\
\hline \multicolumn{7}{|c|}{ Molecular subtype } \\
\hline Luminal A & $44(34.9)$ & $21(67.7)$ & $3(8.3)$ & $6(54.5)$ & $14(29.2)$ & \multirow{4}{*}{$<0.001$} \\
\hline Luminal B & $24(19.0)$ & $5(16.1)$ & $8(22.2)$ & $3(27.3)$ & $8(16.7)$ & \\
\hline HER-2 & $25(19.8)$ & $3(9.7)$ & $12(33.3)$ & $1(9.1)$ & $9(18.8)$ & \\
\hline TNBC & $33(26.2)$ & $2(6.5)$ & $13(36.1)$ & $1(9.1)$ & $17(35.4)$ & \\
\hline Patient death & $41(32.5)$ & $16(51.6)$ & $11(30.6)$ & $4(36.4)$ & $10(20.8)$ & 0.041 \\
\hline
\end{tabular}

ER, estrogen receptor; PR, progesterone receptor; HER-2, human epidermal growth factor-2; LI, labeling index; TNBC, triple negative breast cancer.

contrast, bone metastases are correlated with low histologic grade, ER positivity, ER positivity/progesterone receptor (PR) negativity, strand growth pattern, and the presence of fibrotic tumor stroma $[7,14,15]$. Therefore, it is expected that different metastatic sites would show different expression patterns of PPP-related proteins; however, this has not been well studied.

In the present study, we aimed to analyze the expression of PPP-related proteins at different metastatic sites of metastatic breast cancer and to identify the relationship between protein expression and clinicopathologic factors.

\section{Materials and Methods}

This study was approved by the Institutional Review Board of Severance Hospital. 
TABLE 3: Expression of pentose phosphate pathway-related proteins according to the metastatic site in breast cancer metastases.

\begin{tabular}{|c|c|c|c|c|c|c|}
\hline \multirow{2}{*}{ Parameter } & \multirow{2}{*}{$\begin{array}{c}\text { Total } \\
N=126(\%)\end{array}$} & \multicolumn{4}{|c|}{ Metastatic site } & \multirow{2}{*}{$p$ value } \\
\hline & & $\begin{array}{c}\text { Bone } \\
N=31(\%)\end{array}$ & $\begin{array}{c}\text { Brain } \\
N=36(\%)\end{array}$ & $\begin{array}{c}\text { Liver } \\
N=11(\%)\end{array}$ & $\begin{array}{c}\text { Lung } \\
N=48(\%)\end{array}$ & \\
\hline \multicolumn{7}{|l|}{ G6PDH } \\
\hline Negative & $26(20.6)$ & $7(22.6)$ & $3(8.3)$ & $6(54.5)$ & $10(20.8)$ & \multirow{2}{*}{0.011} \\
\hline Positive & $100(79.4)$ & $24(77.4)$ & $33(91.7)$ & $5(45.5)$ & $38(79.2)$ & \\
\hline \multicolumn{7}{|l|}{ 6PGL } \\
\hline Negative & $89(70.6)$ & $21(67.7)$ & $23(63.9)$ & $11(100.0)$ & $34(70.8)$ & \multirow{2}{*}{0.139} \\
\hline Positive & $37(29.4)$ & $10(32.3)$ & $13(36.1)$ & $0(0.0)$ & $14(29.2)$ & \\
\hline \multicolumn{7}{|l|}{$6 \mathrm{PGDH}$} \\
\hline Negative & $122(96.8)$ & $30(96.8)$ & $34(94.4)$ & $11(100.0)$ & 47 (97.9) & \multirow{2}{*}{0.750} \\
\hline Positive & $4(3.2)$ & $1(3.2)$ & $2(5.6)$ & $0(0.0)$ & $1(2.1)$ & \\
\hline \multicolumn{7}{|c|}{ NRF2 (cytoplasm) } \\
\hline Negative & $113(89.7)$ & $31(100.0)$ & $26(72.2)$ & $11(100.0)$ & 45 (93.8) & \multirow{2}{*}{0.001} \\
\hline Positive & $13(10.3)$ & $0(0.0)$ & $10(27.8)$ & $0(0.0)$ & $3(6.3)$ & \\
\hline \multicolumn{7}{|c|}{ NRF2 (nuclei) } \\
\hline Negative & $110(87.3)$ & $28(90.3)$ & $28(77.8)$ & $11(100.0)$ & $43(89.6)$ & \multirow{2}{*}{0.170} \\
\hline Positive & $16(12.7)$ & $3(9.7)$ & $8(22.2)$ & $0(0.0)$ & $5(10.4)$ & \\
\hline
\end{tabular}

G6PDH, glucose-6-phosphate dehydrogenase; 6PGL, 6-phosphogluconolactonase; 6PGDH, 6-phosphogluconate dehydrogenase; NRF2, nuclear factor erythroid 2- (NF-E2-) related factor 2.

2.1. Patient Selection. Invasive primary breast cancers and their metastases to distant organs (liver, lung, brain, and bone) were retrieved from the data files of the Department of Pathology of Severance Hospital. Only patients with a diagnosis of invasive ductal carcinoma were included. A total of 162 cases were selected with 49 pairs of primary tumors and their metastases. All slides were re-reviewed and pathologic diagnoses were approved by two pathologists (JSK and WJ). The histological grade was assessed using the Nottingham grading system [16].

2.2. Tissue Microarray. Hematoxylin and eosin- (H\&E-) stained tumor samples were mounted on slides, a representative area was selected, and a corresponding spot was marked on the surface of the paraffin block. Using a biopsy needle, the selected area was punched out and a $3 \mathrm{~mm}$ tissue core was placed onto a $6 \times 5$ recipient block. Tissue was extracted from invasive tumors. More than two tissue cores were extracted to minimize extraction bias. Each tissue core was assigned a unique tissue microarray location number that was linked to a database containing other clinicopathologic data.

2.3. Immunohistochemistry. The antibodies used for immunohistochemistry (IHC) in this study are listed in Table 1. IHC was performed with formalin-fixed, paraffin-embedded tissue sections. Briefly, $3 \mu \mathrm{m}$ thick tissue sections from paraffin blocks were deparaffinized, rehydrated with xylene and alcohol solution, and stained using Ventana Discovery XT automated stainer (Ventana Medical System, Tucson, AZ, USA). CC1 buffer (Cell Conditioning 1; citrate buffer $\mathrm{pH}$
6.0, Ventana Medical System) was used for antigen retrieval. Appropriate positive and negative controls were included.

2.4. Interpretation of Immunohistochemical Results. A cutoff value of $1 \%$ or more positively stained nuclei was used to define ER and PR positivity [17]. HER-2 staining was analyzed according to the American Society of Clinical Oncology/College of American Pathologists guidelines using the following categories: $0=$ no immunostaining; $1+=$ weak incomplete membranous staining in less than $10 \%$ of tumor cells; $2+=$ complete membranous staining that is either uniform or weak in at least $10 \%$ of tumor cells; and $3+=$ uniform intense membranous staining in at least $30 \%$ of tumor cells [18]. HER-2 immunostaining was considered positive when strong (3+) membranous staining was observed and was considered negative for tumors with 0 to $1+$ staining. The tumors showing 2+ HER-2 expression were further evaluated for HER-2 amplification by using silver in situ hybridization (SISH).

IHC results were interpreted after multiplying the staining intensity score (negative, 0 ; weak, 1 ; moderate, 2; strong, 3 ) and the proportion of stained cells (negative, $0 ;<30 \%$ stained, $1 ; \geq 30 \%$ stained, 2 ). Scores of 0 and 1 were considered negative, and scores of 2-4 and 5-6 were considered low and high positivity, respectively [19]. The Ki-67 labeling index (LI) was defined as the percentage of nuclear-stained tumor cells.

2.5. Tumor Phenotype Classification. We classified breast cancer phenotypes according to the IHC results for ER, PR, HER-2 and Ki-67, and SISH results for HER-2 as follows 

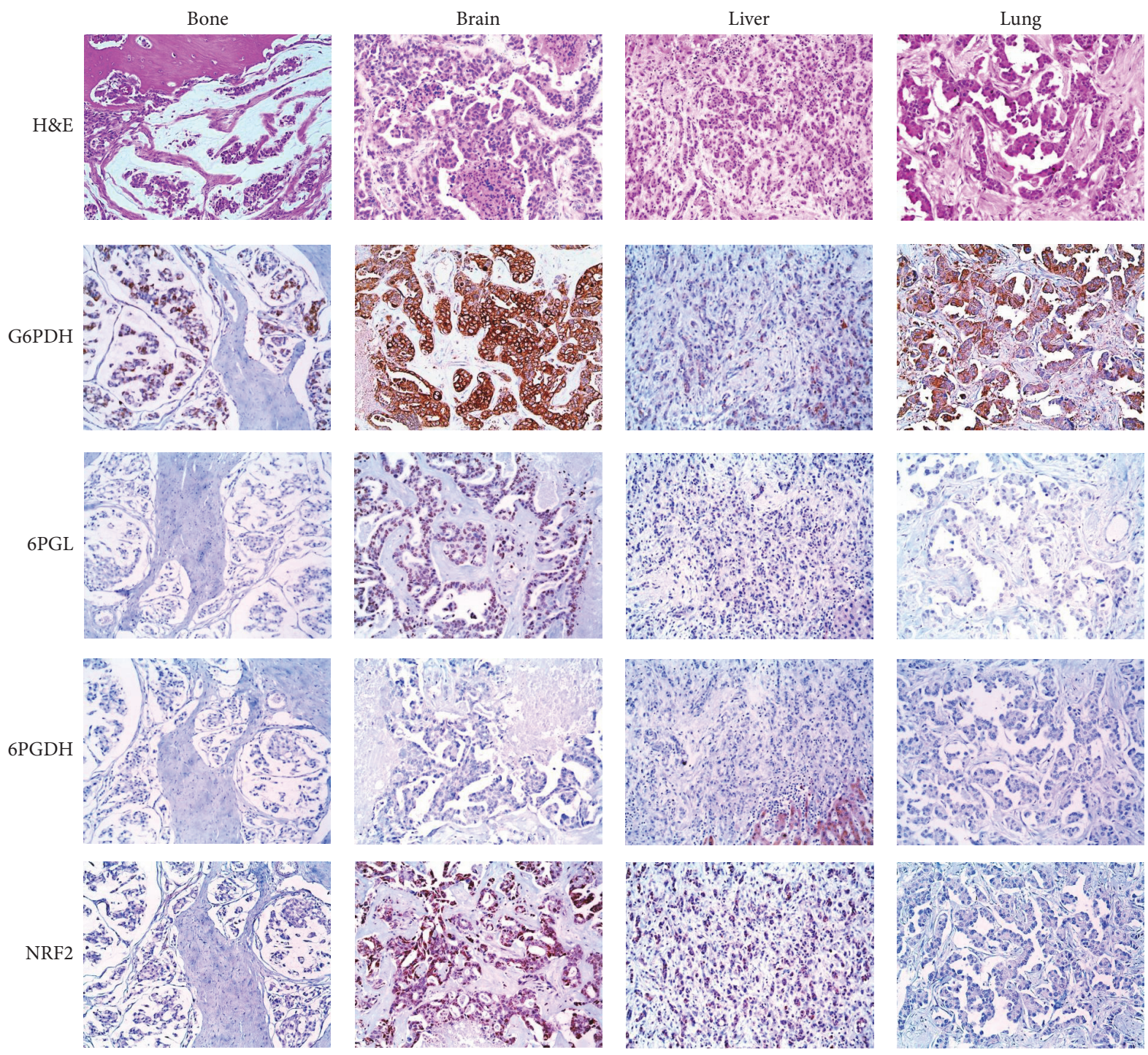

FIGURE 1: Expression of pentose phosphate pathway-related proteins in metastatic breast cancer. The expression of G6PDH and cytoplasmic NRF2 in brain metastases is higher than that at other sites.

[20]: luminal A type: ER or/and PR positive and HER-2 negative and Ki-67 LI <14\%; luminal B type: (HER-2 negative) ER or/and PR positive and HER-2 negative and Ki-67 LI $\geq 14 \%$ and (HER-2 positive) ER or/and PR positive and HER2 overexpressed or/and amplified; HER-2 type: ER and PR negative and HER-2 overexpressed or/and amplified; and triple negative breast cancer (TNBC) type: ER, PR, and HER-2 negative.

2.6. Statistical Analysis. Data were statistically analyzed using SPSS for Windows, version 12.0 (SPSS Inc., Chicago, IL, USA). Correlation analysis of immunostaining results between primary breast cancer and metastatic breast cancer was performed using the McNemar test. Student's $t$-test and Fisher's exact test were used to examine any differences in continuous and categorical variables, respectively. A corrected $p$ value and the Bonferroni method were used for multiple comparisons. Statistical significance was assumed when $p<0.05$. Kaplan-Meier survival curves and log-rank statistics were employed to evaluate time to tumor metastasis and time to survival. Multivariate regression analysis was performed using a Cox proportional hazards model.

\section{Results}

3.1. Clinicopathologic Characteristics of Metastatic Breast Cancer. A total of 126 metastatic breast cancers comprised 31 (24.6\%) bone metastases, 36 (28.6\%) brain metastases, 11 (8.7\%) liver metastases, and 48 (38.1\%) lung metastases (Table 2$)$. ER $(p<0.001), \operatorname{PR}(p<0.001)$, HER-2 $(p=0.032)$, Ki-67 LI $(p=0.008)$, and molecular subtype $(p<0.001)$ differed with regard to the metastatic sites. ER negativity, PR negativity, and HER-2 positivity were more frequent and Ki$67 \mathrm{LI}$ was higher for brain metastases than for the other sites. Predominant molecular subtype was luminal A in bone and 


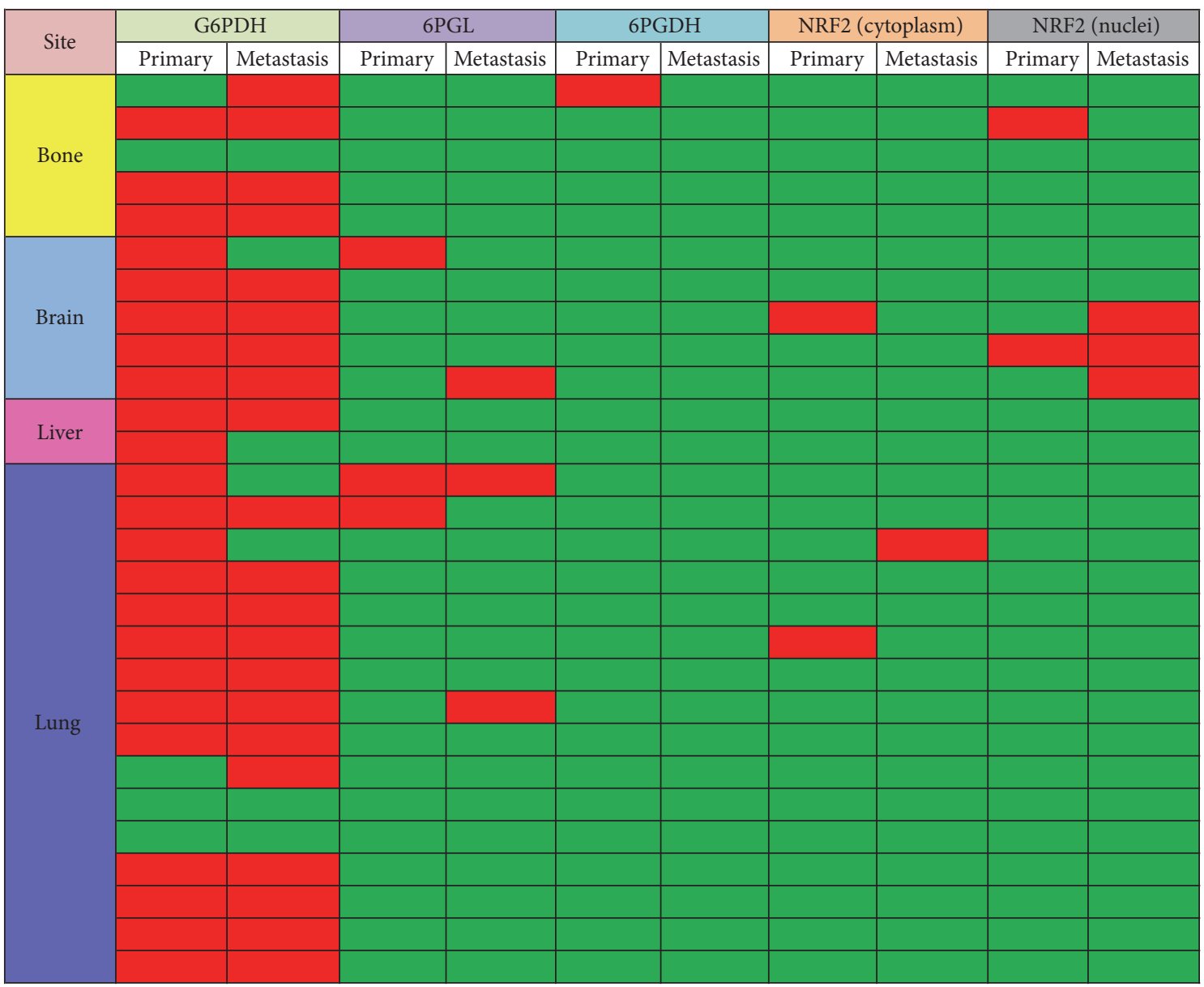

FIGURE 2: Expression status of pentose phosphate pathway-related proteins in paired primary and metastatic breast cancer (red, positive; green, negative).

liver metastases, HER-2 in brain metastases, and TNBC in lung metastases.

3.2. Expression of Pentose Phosphate Pathway-Related Proteins in Metastatic Breast Cancer. G6PDH $(p=0.011)$ and cytoplasmic NRF2 $(p=0.001)$ in metastatic breast cancers were differentially expressed depending on the metastatic sites, with brain metastases showing higher expression of G6PDH and cytoplasmic NRF2 than the other sites (Figure 1 and Table 3). Comparisons of the 28 paired primary metastatic breast cancers revealed differential expression patterns of G6PDH $(n=6,21.4 \%, p=0.688)$, 6PGL $(n=4,14.3 \%, p=$ $1.000), 6 \mathrm{PGDH}(n=1,3.6 \%, p=1.000)$, cytoplasmic NRF2 $(n=3,10.7 \%, p=1.000)$, and nuclear NRF2 $(n=3,10.7 \%$, $p=1.000$ ) (Figure 2). Expression rates of 6PGL, 6PGDH, and cytoplasmic NRF2 were relatively low in metastatic breast cancer and primary breast cancer; the mentioned rates were $29.4 \%, 3.2 \%$, and $10.3 \%$ in metastatic breast cancer and $10.7 \%$, $3.6 \%$, and $7.1 \%$ in primary breast cancer, respectively.

3.3. Correlation between Clinicopathologic Factors and Expression of Pentose Phosphate Pathway-Related Proteins. HER-2 amplification was associated with G6PDH positivity $(p<$
$0.001)$ and cytoplasmic NRF2 positivity $(p=0.015)$. Higher Ki-67 LI was correlated with higher nuclear NRF2 expression ( $p=0.037)$. Luminal B (HER-2 positive) type was associated with G6PDH positivity $(p=0.001)$ (Figure 3 ).

3.4. The Impact of Expression of Pentose Phosphate PathwayRelated Proteins on Prognosis in Metastatic Breast Cancer. Univariate analysis of all metastatic breast cancer cases revealed that the expression of PPP-related proteins had no effect on patient prognosis (Table 4). However, in terms of metastatic sites, expression of 6PGL in bone metastases and 6PGDH in lung metastases was associated with shorter overall survival ( $p=0.040$ and $p=0.002$, resp., Figure 4 ). On multivariate Cox analysis, 6PGL positivity (hazard ratio [HR] 4.180; 95\% confidential interval [CI] 1.160-15.06; $p=0.029$ ) and lower Ki-67 LI (HR 11.853; 95\% CI 1.841-76.30; $p=0.009$ ) were independent risk factors for shorter overall survival in bone metastasis and lung metastasis, respectively (Table 5).

\section{Discussion}

We investigated the expression of PPP-related proteins in metastatic breast cancers and observed differential expression 


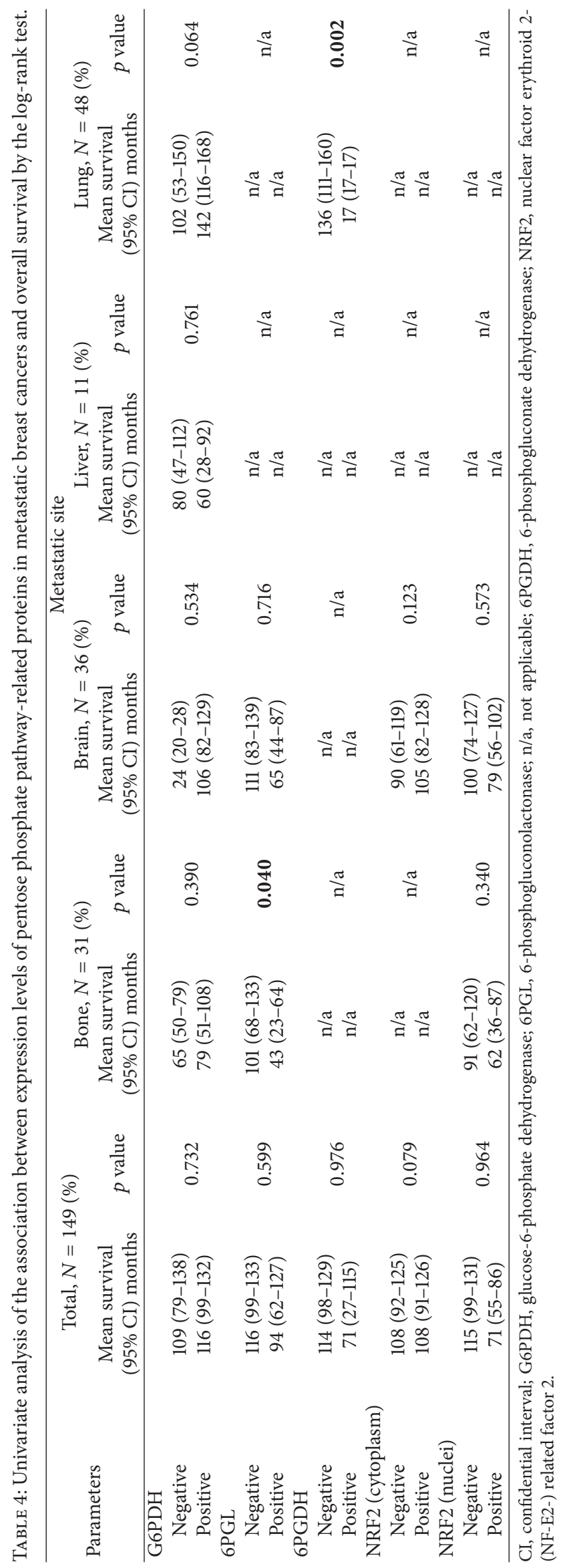



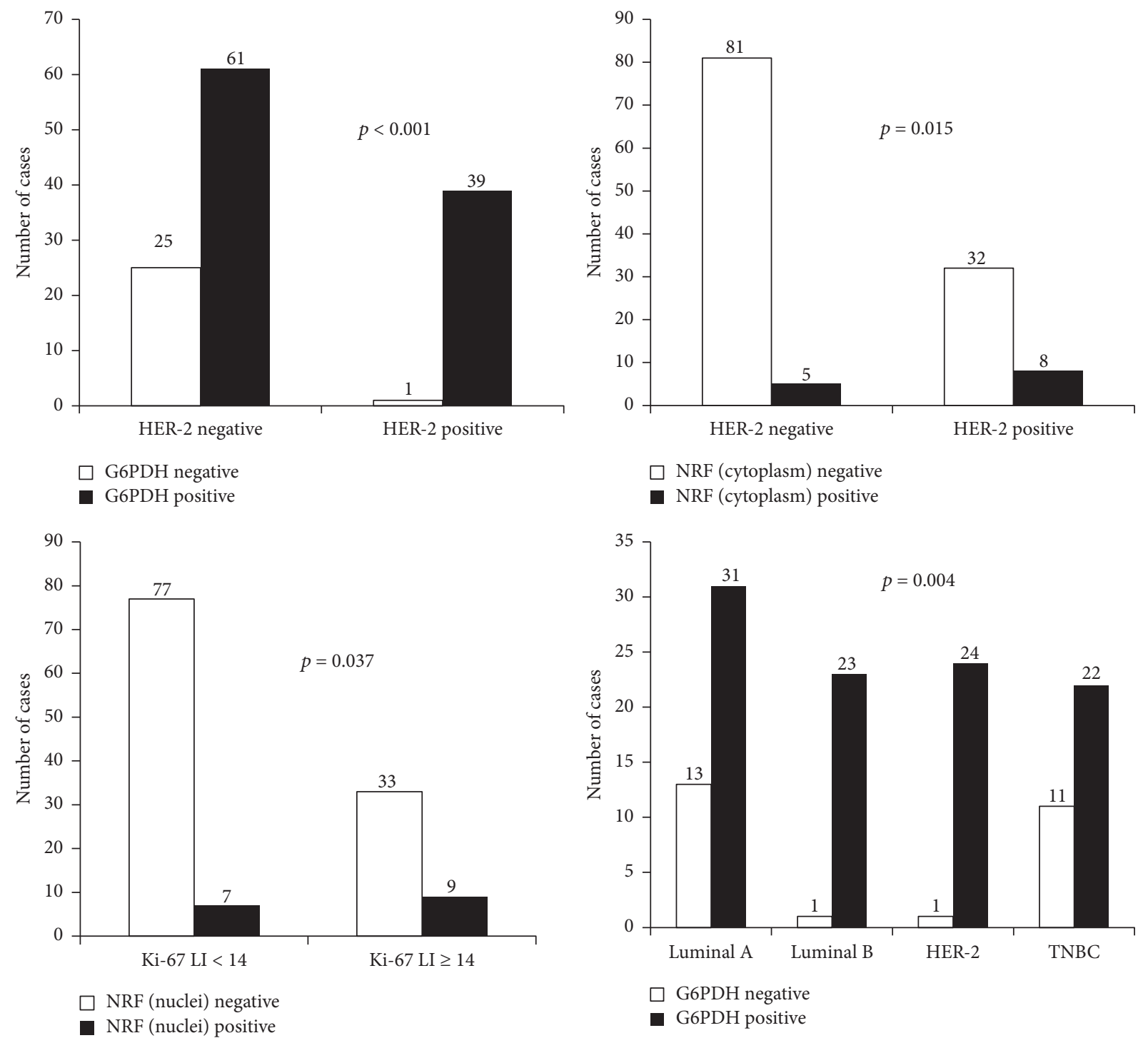

FIGURE 3: Correlation between clinicopathologic factors and expression of pentose phosphate pathway-related proteins.

patterns depending on the metastatic sites. Brain metastases showed higher expression of G6PDH and cytoplasmic NRF2. The site-based variations in the cell biology of metastatic tumors could result in the differential expression of PPPrelated proteins at each metastatic site. In the present study, we found that HER-2 positivity correlated with G6PDH and cytoplasmic NRF2 expression. A previous study in an ErbB2-positive breast cancer cell line BT-474 revealed that knockdown of NRF2 inhibited HER-2 expression [21]. NRF2 is key molecule in the regulation of the PPP and also regulates PPP-related protein expression in tumors [22], which would be affected by specific tumor cells types. Another potential mechanism for differential expression of PPP-related proteins is tumor microenvironment. Various tumor environments could influence the PPP. Compared to the PPP in healthy tissue, the PPP flux is higher in traumatically injured brain tissue $[23,24]$, as well as in brain tumors because of the involvement of NRF2 [22]; thus, an increase in PPP activity is possible in brain metastasis. NRF2 is a nuclear transcription factor that contributes to cellular differentiation, proliferation, and inflammation and that is involved in antioxidant gene activity in neurodegeneration [25] and cardiovascular disease [26]. In human cancers, overexpression of nuclear NRF2 is associated with tumor progression and drug resistance $[27,28]$, and a correlation between nuclear NRF2 expression and higher Ki-67 LI was observed in the present study. Moreover, we found that cytoplasmic NRF2 expression was correlated with HER-2 positivity, mostly in brain metastases. Cytoplasmic expression of NRF2 represents aberrant subcellular localization. In colorectal cancer, cytoplasmic NRF2 expression has been reported to promote cancer cell invasion via regulation of 


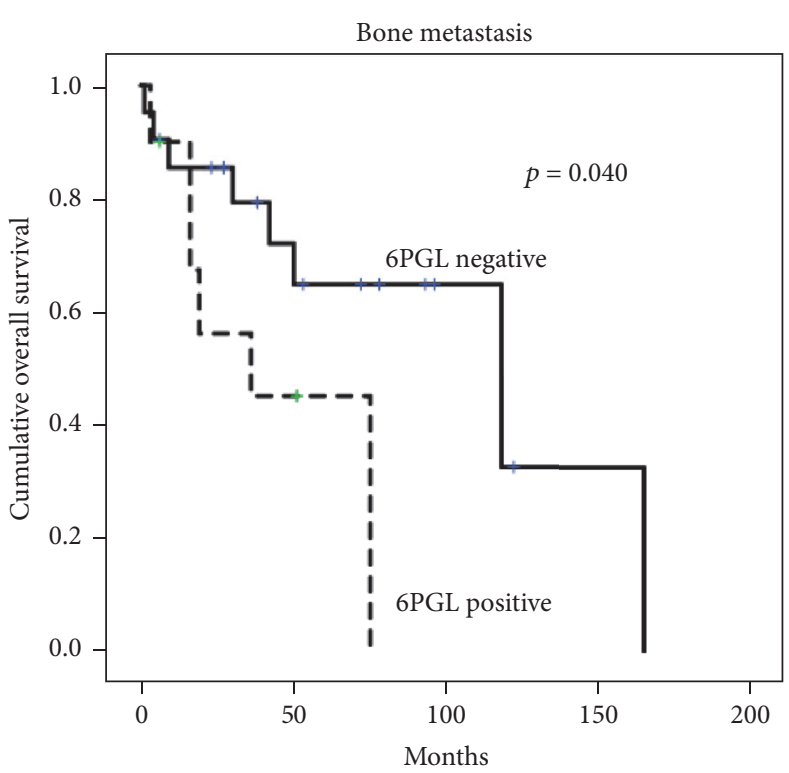

(a)

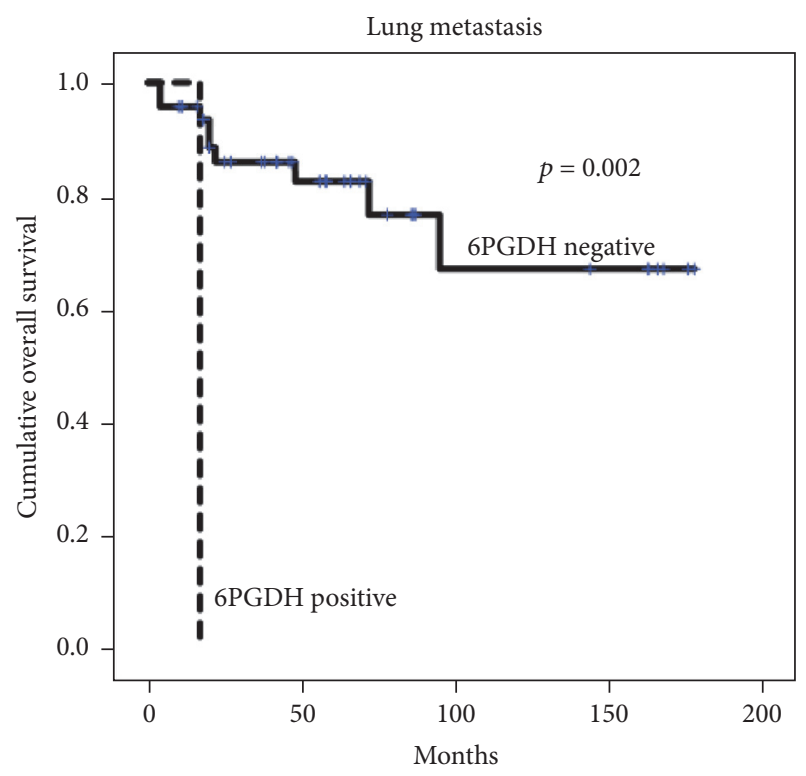

(b)

FIGURE 4: Overall survival according to the expression of pentose phosphate pathway-related proteins in bone metastases (a) and lung metastases (b). Shorter overall survival is associated with 6PGL positivity in bone metastases and 6PGDH positivity in lung metastases.

TABLE 5: Multivariate Cox analysis of the association between expression levels of pentose phosphate pathway-related proteins in metastatic breast cancers and overall survival.

\begin{tabular}{|c|c|c|c|c|c|c|}
\hline \multirow{3}{*}{ Included parameters } & \multicolumn{3}{|c|}{ Bone metastasis } & \multicolumn{3}{|c|}{ Lung metastasis } \\
\hline & \multicolumn{3}{|c|}{ Overall survival } & \multicolumn{3}{|c|}{ Overall survival } \\
\hline & HR & $95 \% \mathrm{CI}$ & $p$ value & HR & $95 \% \mathrm{CI}$ & $p$ value \\
\hline \multicolumn{7}{|l|}{ ER status } \\
\hline Negative versus positive & 1.768 & $0.113-27.61$ & 0.685 & $\mathrm{n} / \mathrm{a}$ & $\mathrm{n} / \mathrm{a}$ & $\mathrm{n} / \mathrm{a}$ \\
\hline \multicolumn{7}{|l|}{ PR status } \\
\hline Negative versus positive & 0.453 & $0.119-1.730$ & 0.247 & $\mathrm{n} / \mathrm{a}$ & $\mathrm{n} / \mathrm{a}$ & $\mathrm{n} / \mathrm{a}$ \\
\hline \multicolumn{7}{|l|}{ HER-2 status } \\
\hline Negative versus positive & 1.025 & $0.217-4.833$ & 0.975 & $\mathrm{n} / \mathrm{a}$ & $\mathrm{n} / \mathrm{a}$ & $\mathrm{n} / \mathrm{a}$ \\
\hline \multicolumn{7}{|l|}{ Ki-67 LI } \\
\hline$\leq 14$ versus $>14$ & 0.961 & $0.031-29.98$ & 0.982 & 11.853 & $1.841-76.30$ & 0.009 \\
\hline \multicolumn{7}{|l|}{ Molecular subtype } \\
\hline TNBC versus non-TNBC & 0.647 & $0.002-254.8$ & 0.886 & $\mathrm{n} / \mathrm{a}$ & $\mathrm{n} / \mathrm{a}$ & $\mathrm{n} / \mathrm{a}$ \\
\hline \multicolumn{7}{|l|}{ 6PGL } \\
\hline Negative versus positive & 4.180 & $1.160-15.06$ & 0.029 & $\mathrm{n} / \mathrm{a}$ & $\mathrm{n} / \mathrm{a}$ & $\mathrm{n} / \mathrm{a}$ \\
\hline \multicolumn{7}{|l|}{$6 \mathrm{PGDH}$} \\
\hline Negative versus positive & $\mathrm{n} / \mathrm{a}$ & $\mathrm{n} / \mathrm{a}$ & $\mathrm{n} / \mathrm{a}$ & 1.362 & $0.130-14.29$ & 0.797 \\
\hline
\end{tabular}

HR, hazard ratio; CI, confidential interval; ER, estrogen receptor; n/a, not applicable; PR, progesterone receptor; HER-2, human epidermal growth factor-2; LI, labeling index; TNBC, triple negative breast cancer; 6PGL, 6-phosphogluconolactonase; n/a, not applicable; 6PGDH, 6-phosphogluconate dehydrogenase.

PSMD4 [29], and a higher frequency of cytoplasmic NRF2 in HER-2-positive cancers and brain metastases might reflect greater invasiveness and aggressiveness.

We found that shorter overall survival was associated with 6PGL positivity in bone metastases and 6PGDH positivity in lung metastases. Expression of PPP-related proteins is associated with poor prognosis in esophageal cancer [30], colon cancer [31], and ocular adnexal tumor [32]. These findings are consistent with our results, suggesting that PPPrelated proteins could be prognostic factors in patients with metastatic breast cancer, especially in patients with bone metastasis. However, further study is required to validate our findings before their application in clinical practice. The results of the present study indicate that PPP-related proteins could be a potential therapeutic target in metastatic breast cancer, particularly for brain metastases, which had a higher 
expression of PPP-related proteins. In previous studies, inhibition of PPP-related proteins induced growth suppression and cell death in leukemia [33], ovary cancer [34], urinary bladder cancer [35], and breast and prostate cancer [36], which suggested that control of expression of PPP-related proteins could be an effective treatment strategy. Therefore, further development of PPP-related protein targeting agent should be evaluated in metastatic breast cancer patients through clinical trials. In conclusion, PPP-related proteins in metastatic breast cancer showed different expression patterns that were specific to the metastatic sites, with increased expression in brain metastases. Expression of PPP-related proteins at specific metastatic sites correlated with prognosis.

\section{Competing Interests}

The authors declare that they have no competing interests.

\section{References}

[1] C. Riganti, E. Gazzano, M. Polimeni, E. Aldieri, and D. Ghigo, "The pentose phosphate pathway: an antioxidant defense and a crossroad in tumor cell fate," Free Radical Biology and Medicine, vol. 53, no. 3, pp. 421-436, 2012.

[2] C. Zhang, Z. Zhang, Y. Zhu, and S. Qin, "Glucose-6-phosphate dehydrogenase: a biomarker and potential therapeutic target for cancer," Anti-Cancer Agents in Medicinal Chemistry, vol. 14, no. 2, pp. 280-289, 2014.

[3] G. Lucarelli, V. Galleggiante, M. Rutigliano et al., "Metabolomic profile of glycolysis and the pentose phosphate pathway identifies the central role of glucose-6-phosphate dehydrogenase in clear cell-renal cell carcinoma," Oncotarget, vol. 6, no. 15, pp. 13371-13386, 2015.

[4] E. C. Woodhouse, R. F. Chuaqui, and L. A. Liotta, "General mechanisms of metastasis," Cancer, vol. 80, S8, pp. 1529-1537, 1997.

[5] R. J. Weil, D. C. Palmieri, J. L. Bronder, A. M. Stark, and P. S. Steeg, "Breast cancer metastasis to the central nervous system," American Journal of Pathology, vol. 167, no. 4, pp. 913-920, 2005.

[6] H. Abali and I. Çelik, "High incidence of central nervous system involvement in patients with breast cancer treated with epirubicin and docetaxel," American Journal of Clinical Oncology, vol. 25, no. 6, pp. 632-633, 2002.

[7] M. Colleoni, A. O’Neill, A. Goldhirsch et al., "Identifying breast cancer patients at high risk for bone metastases," Journal of Clinical Oncology, vol. 18, no. 23, pp. 3925-3925, 2000.

[8] A. J. Evans, J. J. James, E. J. Cornford et al., "Brain metastases from breast cancer: identification of a high-risk group," Clinical Oncology (Royal College of Radiologists), vol. 16, no. 5, pp. 345349, 2004.

[9] D. G. Hicks, S. M. Short, N. L. Prescott et al., "Breast cancers with brain metastases are more likely to be estrogen receptor negative, express the basal cytokeratin CK5/6, and overexpress HER2 or EGFR," American Journal of Surgical Pathology, vol. 30, no. 9, pp. 1097-1104, 2006.

[10] T. Lorincz, J. Tóth, G. Badalian, J. Tímár, and M. Szendroi, "HER-2/neu genotype of breast cancer may change in bone metastasis," Pathology and Oncology Research, vol. 12, no. 3, pp. 149-152, 2006.
[11] J. Gaedcke, F. Traub, S. Milde et al., "Predominance of the basal type and HER-2/neu type in brain metastasis from breast cancer," Modern Pathology, vol. 20, no. 8, pp. 864-870, 2007.

[12] G. L. Nicolson, "Organ specificity of tumor metastasis: role of preferential adhesion, invasion and growth of malignant cells at specific secondary sites," Cancer and Metastasis Review, vol. 7, no. 2, pp. 143-188, 1988.

[13] S. Paget, "The distribution of secondary growths in cancer of the breast," The Lancet, vol. 133, no. 3421, pp. 571-573, 1889.

[14] T. Hasebe, S. Imoto, T. Yokose, G.-I. Ishii, M. Iwasaki, and N. Wada, "Histopathologic factors significantly associated with initial organ-specific metastasis by invasive ductal carcinoma of the breast: a prospective study," Human Pathology, vol. 39, no. 5, pp. 681-693, 2008.

[15] B. Wei, J. Wang, P. Bourne et al., "Bone metastasis is strongly associated with estrogen receptor-positive/progesterone receptor-negative breast carcinomas," Human Pathology, vol. 39, no. 12, pp. 1809-1815, 2008.

[16] C. W. Elston and I. O. Ellis, "Pathological prognostic factors in breast cancer. I. The value of histological grade in breast cancer: experience from a large study with long-term followup," Histopathology, vol. 19, no. 5, pp. 403-410, 1991.

[17] Hammond, "American Society of Clinical Oncology/College of American Pathologists guideline recommendations for immunohistochemical testing of estrogen and progesterone receptors in breast cancer," Journal of Clinical Oncology, vol. 28, no. 16, pp. 2784-2795, 2010.

[18] A. C. Wolff, M. E. H. Hammond, J. N. Schwartz et al., "American Society of Clinical Oncology/College of American Pathologists guideline recommendations for human epidermal growth factor receptor 2 testing in breast cancer," Journal of Clinical Oncology, vol. 25, no. 1, pp. 118-145, 2007.

[19] K. Y. Won, G. Y. Kim, Y. W. Kim, J. Y. Song, and S.-J. Lim, "Clinicopathologic correlation of beclin-1 and bcl-2 expression in human breast cancer," Human Pathology, vol. 41, no. 1, pp. 107-112, 2010.

[20] A. Goldhirsch, W. C. Wood, A. S. Coates, R. D. Gelber, B. Thürlimann, and H.-J. Senn, "Strategies for subtypes-dealing with the diversity of breast cancer: Highlights of the St Gallen international expert consensus on the primary therapy of early breast cancer 2011," Annals of Oncology, vol. 22, no. 8, pp. 17361747, 2011.

[21] S. Manandhar, B.-H. Choi, K.-A. Jung et al., "NRF2 inhibition represses ErbB2 signaling in ovarian carcinoma cells: implications for tumor growth retardation and docetaxel sensitivity," Free Radical Biology and Medicine, vol. 52, no. 9, pp. 1773-1785, 2012.

[22] F. Ahmad, D. Dixit, V. Sharma et al., "Nrf2-driven TERT regulates pentose phosphate pathway in glioblastoma," Cell Death \& Disease, vol. 7, Article ID e2213, 2016.

[23] B. Polásková and E. Slezáková, "Our experience with reeducation in aphasia," Acta Universitatis Palackianae Olomucensis Facultatis Medicae, vol. 121, pp. 179-187, 1989.

[24] J. R. Dusick, T. C. Glenn, W. N. P. Lee et al., "Increased pentose phosphate pathway flux after clinical traumatic brain injury: a $\left[1,2-{ }^{13} \mathrm{C}_{2}\right]$ glucose labeling study in humans," Journal of Cerebral Blood Flow and Metabolism, vol. 27, no. 9, pp. 1593-1602, 2007.

[25] C. P. Ramsey, C. A. Glass, M. B. Montgomery et al., "Expression of Nrf2 in neurodegenerative diseases," Journal of Neuropathology and Experimental Neurology, vol. 66, no. 1, pp. 75-85, 2007. 
[26] S. Jiang, Y. Yang, T. Li et al., "An overview of the mechanisms and novel roles of Nrf2 in cardiovascular diseases," Expert Opinion on Therapeutic Targets, vol. 20, no. 12, pp. 1413-1424, 2016.

[27] S. Homma, Y. Ishii, Y. Morishima et al., "Nrf2 enhances cell proliferation and resistance to anticancer drugs in human lung cancer," Clinical Cancer Research, vol. 15, no. 10, pp. 3423-3432, 2009.

[28] H. J. Kang, Y. W. Yi, Y. B. Hong et al., "HER2 confers drug resistance of human breast cancer cells through activation of NRF2 by direct interaction," Scientific Reports, vol. 4, article 7201, 2014.

[29] P.-L. Lin, J. T. Chang, D.-W. Wu, C.-C. Huang, and H. Lee, "Cytoplasmic localization of Nrf2 promotes colorectal cancer with more aggressive tumors via upregulation of PSMD4," Free Radical Biology and Medicine, vol. 95, pp. 121-132, 2016.

[30] X. Wang, X. Li, X. Zhang et al., "Glucose-6-phosphate dehydrogenase expression is correlated with poor clinical prognosis in esophageal squamous cell carcinoma," European Journal of Surgical Oncology, vol. 41, no. 10, pp. 1293-1299, 2015.

[31] K. Ahopelto, C. Böckelman, J. Hagström, S. Koskensalo, and C. Haglund, "Transketolase-like protein 1 expression predicts poor prognosis in colorectal cancer," Cancer Biology and Therapy, vol. 17, no. 2, pp. 163-168, 2016.

[32] C. A. Lange, J. Tisch-Rottensteiner, D. Böhringer, G. Martin, J. Schwartzkopff, and C. Auw-Haedrich, "Enhanced TKTL1 expression in malignant tumors of the ocular adnexa predicts clinical outcome," Ophthalmology, vol. 119, no. 9, pp. 1924-1929, 2012.

[33] S. Elf, R. Lin, S. Xia et al., "Targeting 6-phosphogluconate dehydrogenase in the oxidative PPP sensitizes leukemia cells to antimalarial agent dihydroartemisinin," Oncogene, vol. 36, no. 2, pp. 254-262, 2017.

[34] D. Catanzaro, E. Gaude, G. Orso et al., "Inhibition of glucose-6phosphate dehydrogenase sensitizes cisplatin-resistant cells to death," Oncotarget, vol. 6, no. 30, pp. 30102-30114, 2015.

[35] X. Wang, G. Wu, G. Cao et al., "Zoledronic acid inhibits the pentose phosphate pathway through attenuating the RasTAp73-G6PD axis in bladder cancer cells," Molecular Medicine Reports, vol. 12, no. 3, pp. 4620-4625, 2015.

[36] L. Li, M. A. Fath, P. M. Scarbrough, W. H. Watson, and D. R. Spitz, "Combined inhibition of glycolysis, the pentose cycle, and thioredoxin metabolism selectively increases cytotoxicity and oxidative stress in human breast and prostate cancer," Redox Biology, vol. 4, pp. 127-135, 2015. 


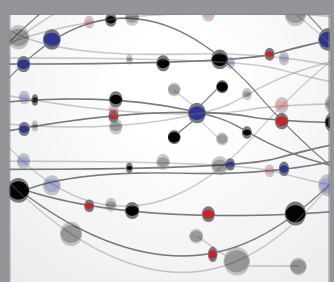

The Scientific World Journal
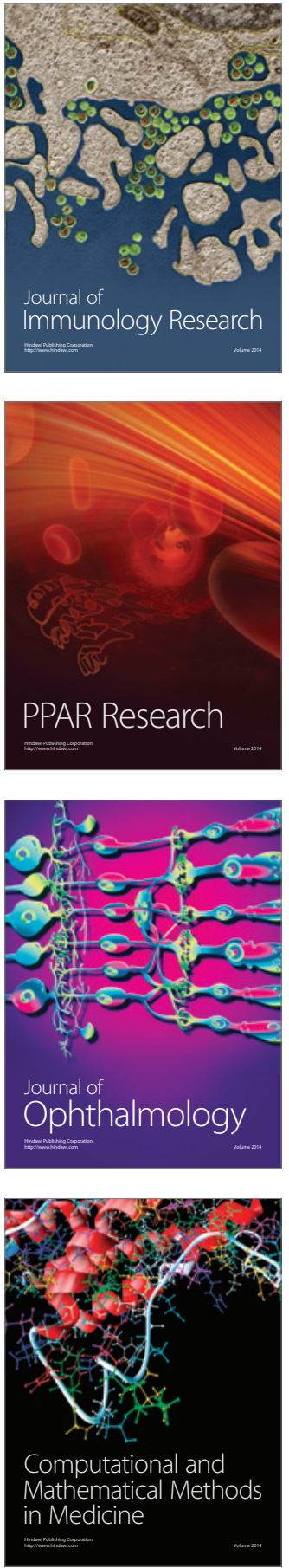

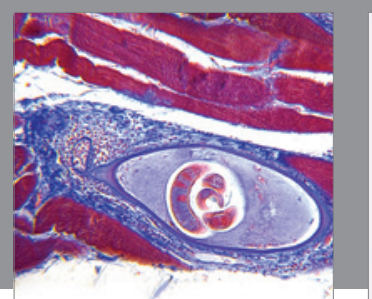

Gastroenterology Research and Practice
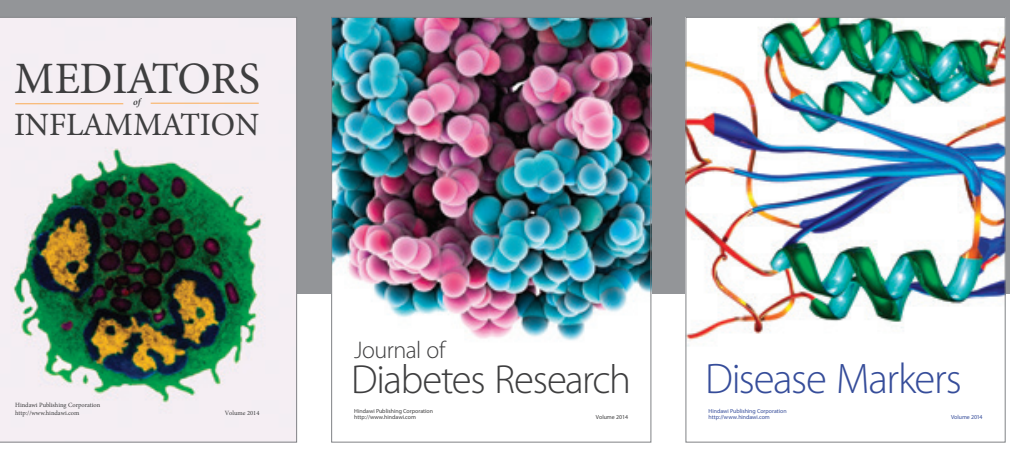

Disease Markers

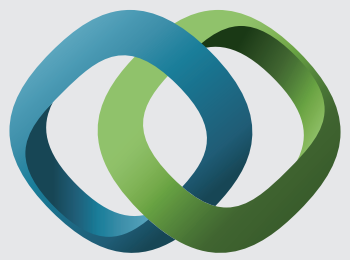

\section{Hindawi}

Submit your manuscripts at

https://www.hindawi.com
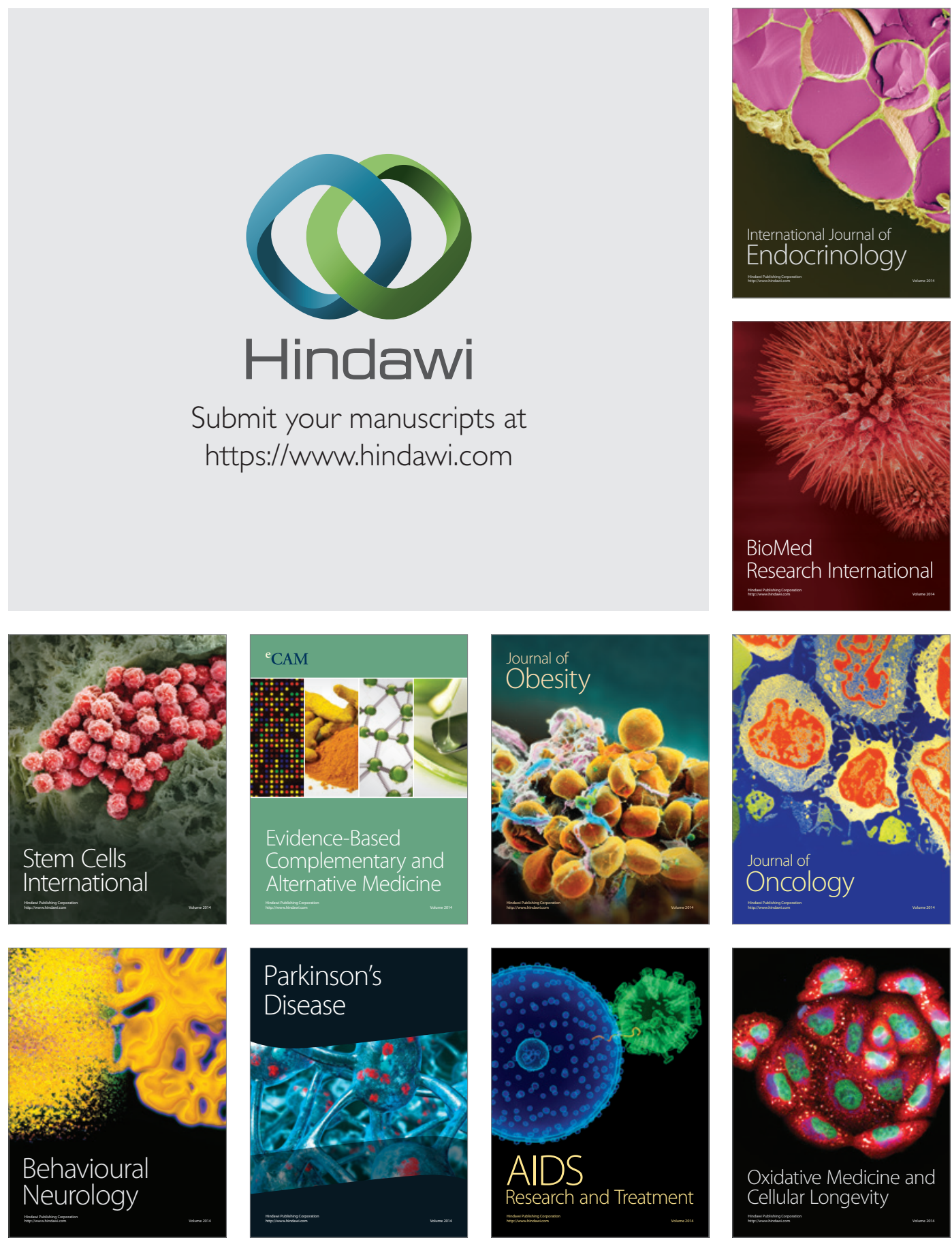